\title{
Nonlinear dynamics of a marine phytoplankton-zooplankton system
}

\author{
Pengfei Wang ${ }^{1,2}$, Min Zhao ${ }^{2,3^{*}}$, Hengguo Yu ${ }^{2,3}$, Chuanjun Dai ${ }^{2,3}$, Nan Wang ${ }^{1,2}$ and Beibei Wang ${ }^{1,2}$
}

\footnotetext{
"Correspondence: zmcn@tom.com ${ }^{2}$ Zhejiang Provincial Key Laboratory for Water Environment and Marine Biological Resources Protection, Wenzhou University, Wenzhou, Zhejiang 325035, P.R. China

${ }^{3}$ School of Life and Environmental Science, Wenzhou University, Wenzhou, Zhejiang 325035, P.R. China

Full list of author information is available at the end of the article
}

\begin{abstract}
In the paper, the nonlinear dynamics of a marine phytoplankton-zooplankton system is discussed in detail. The aim is to deeply expound how to use the dynamical laws of marine ecological system to perfectly reflect the operating rules of marine ecosystems. The mathematical work has been pursuing the investigation of some conditions for the local and global stability of the equilibrium, as well as Turing instability, which can deduce a key parameter control relationship and in turn can provide a theoretical basis for the numerical simulation. The simulation work indicates that the theoretical results are correct, and the nonlinear dynamics of the marine system mainly depend on some critical parameters. The results further suggested that the diffusion and the environmental carrying capacity have an important role to play in the interaction between phytoplankton and zooplankton. All these results are expected to be of use in the study of the internal operating rules and characteristics of marine ecosystems.
\end{abstract}

Keywords: dynamics; pattern formation; reaction-diffusion; stability

\section{Introduction}

It is well known that plankton systems are abundant in marine ecosystems, where they comprise phytoplankton and zooplankton. Phytoplankton are primary producers in the marine ecosystem, where they form the basis of the food chain. Phytoplankton systems also play very important roles in chemical cycles and energy conversion marine ecosystems. Zooplankton can control the amount of phytoplankton via their consumption, where the zooplankton systems then provide a resource that is utilized by consumers at higher trophic levels. Thus, plankton research is highly significant for fishery production and basic theoretical marine science, further the importance of plankton for ecosystems is gradually recognized by contemporary researchers [1].

In marine ecosystems, animal populations often migrate to other habitats to search for food, widen their gene pool, or extend their territory. Factors such as latitude, salinity, ocean currents, and light can also affect their distribution. Thus, the dynamic behaviors present in the trophic interactions of aquatic ecosystems are highly complex. However, it is difficult to describe the dynamics of plankton ecosystems. In recent years, major progress has been made in the mathematical modeling of ecosystems and many studies have described the different dynamical behaviors of marine ecosystems [2-5]. In general, these systems are all predator-prey systems. Some previous studies have provided detailed

(c) 2016 Wang et al. This article is distributed under the terms of the Creative Commons Attribution 4.0 International License (http://creativecommons.org/licenses/by/4.0/), which permits unrestricted use, distribution, and reproduction in any medium, provided you give appropriate credit to the original author(s) and the source, provide a link to the Creative Commons license, and indicate if changes were made. 
analyses of these predator-prey systems [6-10]. There have been numerous investigations of the dynamics of phytoplankton-zooplankton models [11-13]. For example, Steele [13] considered the interactions among phytoplankton and herbivore. References [12, 14-16] incorporated nutrients into the interactions among plankton. The toxic substances produced by phytoplankton have a major impact on phytoplankton-zooplankton interactions, there have been several analyses of toxic-phytoplankton systems [17-21]. In [22], a mathematical system was proposed to describe the interaction between non-toxic and toxic phytoplankton in the presence of a single nutrient. In many ways research into plankton is still highly active.

Mathematical systems and methods are often used to study ecological problems [6, 7, $23,24]$. However, some of the mathematical systems used to describe ecological phenomena cannot be applied to planktonic systems. Thus, we need to select specific functions and functional responses to build a model of a planktonic system. In 1952, Turing [25] proposed the reaction-diffusion equation and described the concept of Turing instability. The importance of pattern formation was studied subsequently by Segal and Jackson [26]. Reaction-diffusion processes are now very important for describing the dynamic behavior of phytoplankton-zooplankton systems. In 1965, Holling [27] proposed three types of biological functional responses, called Holling types I, II, and III. In [28], analysis was provided of reaction-diffusion toxin-phytoplankton-zooplankton systems with either Holling-type II or Holling-type III functional responses. In [29], an analysis was presented of a predator-prey system with a Holling-type IV functional response. In 1965, Ivlev [30] proposed the Ivlev-type functional response. Subsequent studies also considered a grazing function as Ivlev-type function in a prey-predator system [31, 32].

In the present study, we consider a two-component phytoplankton-zooplankton model, which can be described based on the following assumptions: First, it is assumed that the conditions of phytoplankton growth are ideal, thus the phytoplankton population follows logistical growth in the absence of zooplankton. Second, a direct link between the phytoplankton population and zooplankton population cannot be represented unless quantitative methods are applied; the previous work clearly shows that the grazing relationship of zooplankton population on phytoplankton population may follow Holling II response curve in realistic ecosystem, which has been studied by many ecologists, for example, Fussmann et al. have used a Holling II functional response to model the interaction of zooplankton population and phytoplankton population, and the modeling results can well agreed with experimental results [33]. Thus we apply a Holling II functional response to describe their relationship between zooplankton population and phytoplankton population with sufficient accuracy in this paper. Finally, it is assumed that zooplankton population has sufficient resources for alternative food, thus we applied the logistic growth law to describe this characteristic of zooplankton population in the case of extinction of the phytoplankton. This is because alternative food sources may have an important role to play in promoting the persistence of zooplankton-phytoplankton systems [34]. Based on the above assumptions, we can propose the following system:

$$
\left\{\begin{array}{l}
\frac{d P}{d t}=r_{1} P\left(1-\frac{P}{K_{1}}\right)-\frac{\beta_{1} P Z}{m+P}, \\
\frac{d Z}{d t}=r_{2} Z\left(1-\frac{Z}{K_{2}}\right)+\frac{\beta_{2} P Z}{m+P},
\end{array}\right.
$$


where $P$ is the density of the phytoplankton population and $Z$ is the density of the zooplankton population at time $t$; the seven parameters $r_{1}, K_{1}, m, \beta_{1}, \beta_{2}, r_{2}, K_{2}$ are positive constants; $r_{1}, K_{1}$ are the intrinsic growth rate and environmental carrying capacity of the phytoplankton, respectively. $m$ is the half saturation constant for a Holling-type II functional response, $\beta_{1}$ is the rate of predation of zooplankton, and $\beta_{2}$ denotes the ratio of biomass consumed by zooplankton during its growth.

In marine ecosystems, turbulent diffusion can also cause the movement of plankton. Thus, this diffusion should also be considered in plankton models. We assume that no plankton species enter or leave the water at the boundary. If we consider the evolution of the plankton model using constant diffusion coefficients, then system (1.1) can be reduced to the following system:

$$
\left\{\begin{array}{l}
\frac{\partial P}{\partial t}=r_{1} P\left(1-\frac{P}{K_{1}}\right)-\frac{\beta_{1} P Z}{m+P}+D_{1} \Delta P \\
\frac{\partial Z}{\partial t}=r_{2} Z\left(1-\frac{Z}{K_{2}}\right)+\frac{\beta_{2} P Z}{m+P}+D_{2} \Delta Z
\end{array}\right.
$$

where $D_{1}$ and $D_{2}$ are the constant diffusion coefficients of the phytoplankton and zooplankton, respectively. We take the boundary condition of the plankton population $P(s, t)$ and $Z(s, t)$ in $s \in \Omega, t>0$, as follows:

$$
\frac{\partial P}{\partial n}=\frac{\partial Z}{\partial n}=0, \quad s \in \partial \Omega, t>0,
$$

and the initial conditions are

$$
P(0, s)=P_{0}(s)>0, \quad Z(0, s)=Z_{0}(s)>0, \quad s \in \Omega .
$$

The equilibrium states of system (1.2) are the same as those of the homogeneous system (1.1).

Based on the above considerations, the remainder of this paper is organized as follows. In Section 2, we study the equilibrium of the system and the stability of the system. In Section 3, we consider the system with diffusion. We derive the criteria for diffusive stability and the Turing instability region of the system. Finally, simulations and discussions are presented.

\section{Equilibrium and the stability of system (1.1)}

\subsection{Equilibrium of the system}

Let $f(P, Z)$ and $g(P, Z)$ be the right hand sides of the equations in (1.1). It is obvious that the equilibria $E_{0}=(0,0), E_{1}=\left(K_{1}, 0\right)$, and $E_{2}=\left(0, K_{2}\right)$ always exist. By $f(P, Z)=0$, we can obtain the vertical isoclines $l_{1}: Z=-\frac{r_{1}}{K_{1} \beta_{1}}\left(P^{2}+\left(m-K_{1}\right) P-K_{1} m\right)$, and it is a downwards parabola. The axis of symmetry is $P=\frac{K_{1}-m}{2}$, and we have the $y$ axis by $Z=\frac{r_{1} m}{\beta_{1}}$. By $g(P, Z)=$ 0 , we can obtain the horizontal isoclines $l_{2}: Z=K_{2}+\frac{\beta_{2} K_{2} P}{r_{2}(m+P)}$. It is easy to see that the line $P=$ $-m$ is the asymptote of $l_{2}$. We can see that $l_{2}$ increases monotonically when $P \in(-m,+\infty)$, and we have the $y$ axis by $Z=K_{2}$. Next we discuss the existence of positive equilibria.

(i) If $K_{1}>m, \frac{r_{1} m}{\beta_{1}} \leq K_{2} \leq \frac{r_{1} r_{2}\left(K_{1}+m\right)^{3}}{4 K_{1} \beta_{1}\left[r_{2}\left(K_{1}+m\right)+\beta_{2}\left(K_{1}-m\right)\right]}$, then there exist two positive equilibria $E_{e 1}=\left(P_{e 1}, Z_{e 1}\right)$ and $E_{e 2}=\left(P_{e 2}, Z_{e 2}\right)$ in system (1.1), where $P_{e 1}<\frac{K_{1}-m}{2}, P_{e 2}>\frac{K_{1}-m}{2}$.

(ii) If $K_{1}>m, \frac{r_{1} r_{2}\left(K_{1}+m\right)^{3}}{4 K_{1} \beta_{1}\left[r_{2}\left(K_{1}+m\right)+\beta_{2}\left(K_{1}-m\right)\right]}<K_{2}<\frac{r_{1} m}{\beta_{1}}$, then there exists one positive equilibrium $E_{e 3}=\left(P_{e 3}, Z_{e 3}\right)$ in system (1.1), where $P_{e 3}<\frac{K_{1}-m}{2}$. 
(iii) If (1) $K_{1}>m, K_{2}<\min \left\{\frac{r_{1} m}{\beta_{1}}, \frac{r_{1} r_{2}\left(K_{1}+m\right)^{3}}{4 K_{1} \beta_{1}\left[r_{2}\left(K_{1}+m\right)+\beta_{2}\left(K_{1}-m\right)\right]}\right\}$, or (2) $K_{1}<m, K_{2}<\frac{r_{1} m}{\beta_{1}}$, then there at least exists one positive equilibrium $E_{e 4}=\left(P_{e 4}, Z_{e 4}\right)$ in system $(1.1)$, where $P_{e 4}>\frac{K_{1}-m}{2}$.

\subsection{Stability of the equilibrium}

Next, we discuss the local behaviors of the equilibria of system (1.1). The Jacobian matrix takes the following form:

$$
\left(\begin{array}{cc}
r_{1}\left(1-\frac{P}{K_{1}}\right)-\frac{r_{1}}{K_{1}} P-\frac{\beta_{1} m Z}{(m+P)^{2}} & -\frac{\beta_{1} P}{m+P} \\
\frac{\beta_{2} K_{2} Z}{(m+P)^{2}} & r_{2}\left(1-\frac{Z}{K_{2}}\right)-\frac{r_{2}}{K_{2}} Z+\frac{\beta_{2} P}{m+P}
\end{array}\right) .
$$

It is obvious that $E_{0}=(0,0)$ is unstable and $E_{1}=\left(K_{1}, 0\right)$ is a saddle point. $E_{2}=\left(0, K_{2}\right)$ is a saddle point if $r_{1}>\frac{\beta_{1} K_{2}}{m}$, and is locally asymptotically stable when $r_{1}<\frac{\beta_{1} K_{2}}{m}$.

We now analyze the local stability of the positive equilibria. Let $\bar{E}=(\bar{P}, \bar{Z})$ be an arbitrary positive equilibrium. We can obtain

$$
\left\{\begin{array}{l}
r_{1}\left(1-\frac{\bar{P}}{K_{1}}\right)-\frac{\beta_{1} \bar{Z}}{m+\bar{P}}=0 \\
r_{2}\left(1-\frac{\bar{Z}}{K_{2}}\right)+\frac{\beta_{2} \bar{P}}{m+\bar{P}}=0
\end{array}\right.
$$

so the Jacobian matrix for $\bar{E}=(\bar{P}, \bar{Z})$ is given by

$$
J_{\bar{E}}=\left(\begin{array}{cc}
\bar{P}\left[\frac{r_{1}}{m+\bar{P}}\left(1-\frac{\bar{P}}{K_{1}}\right)-\frac{r_{1}}{K_{1}}\right] & -\frac{\beta_{1} \bar{P}}{m+\bar{P}} \\
\frac{\beta_{2} K_{2} \bar{Z}}{(m+\bar{P})^{2}} & -\frac{r_{2} \bar{Z}}{K_{2}}
\end{array}\right)=\left(\begin{array}{ll}
J_{11} & J_{12} \\
J_{21} & J_{22}
\end{array}\right) .
$$

Note that

$$
\begin{aligned}
& \operatorname{tr}\left(J_{\bar{E}}\right)=\bar{P}\left[\frac{r_{1}}{m+\bar{P}}\left(1-\frac{\bar{P}}{K_{1}}\right)-\frac{r_{1}}{K_{1}}\right]-\frac{r_{2} \bar{Z}}{K_{2}}, \\
& \operatorname{det}\left(J_{\bar{E}}\right)=-\bar{P}\left[\frac{r_{1}}{m+\bar{P}}\left(1-\frac{\bar{P}}{K_{1}}\right)-\frac{r_{1}}{K_{1}}\right] \frac{r_{2} \bar{Z}}{K_{2}}+\frac{\beta_{2} K_{2} \bar{Z}}{(m+\bar{P})^{2}} \frac{\beta_{1} \bar{P}}{m+\bar{P}} .
\end{aligned}
$$

If $\bar{P}>\frac{K_{1}-m}{2}$, we can obtain $J_{11}<0$, thus $\operatorname{tr}\left(J_{\bar{E}}\right)<0$ and $\operatorname{det}\left(J_{\bar{E}}\right)>0$. The equilibrium $\bar{E}=(\bar{P}, \bar{Z})$ is always locally asymptotically stable. If $\bar{P}<\frac{K_{1}-m}{2}$, we can obtain $J_{11}>0$. Hence, based on the above analysis, we have the following results.

Lemma 2.1 If there exists one positive equilibrium $E_{e 4}=\left(P_{e 4}, Z_{e 4}\right)$ in system $(1.1)$ and $P_{e 4}>$ $\frac{K_{1}-m}{2}$, then the equilibrium is always locally asymptotically stable.

Lemma 2.2 If there exist two positive equilibria $E_{e 1}=\left(P_{e 1}, Z_{e 1}\right)$ and $E_{e 2}=\left(P_{e 2}, Z_{e 2}\right)$ in system (1.1), we find that $E_{e 2}=\left(P_{e 2}, Z_{e 2}\right)$ is always locally asymptotically stable, and $E_{e 1}=$ $\left(P_{e 1}, Z_{e 1}\right)$ is a saddle point if $K_{2} \leq m$.

Proof Define $F(P)=-\frac{r_{1}}{K_{1} \beta_{1}}\left[P^{2}+\left(m-K_{1}\right) P-K_{1} m\right]-\left[K_{2}+\frac{\beta_{2} K_{2} P}{r_{2}(m+P)}\right]$ where $0<P<\frac{K_{1}-m}{2}$. By combining this with the conditions in (i) we find that $F^{\prime}(P)>0$ when $0<P<\frac{K_{1}-m}{2}$. 
Hence, the inequality of $\frac{\beta_{1} \beta_{2} K_{2}}{(m+P)^{2}}<-\frac{r_{1} r_{2}}{m K_{1}}\left(m+2 P-K_{1}\right)\left(0<P<\frac{K_{1}-m}{2}\right)$ is established. Therefore

$$
\begin{aligned}
\operatorname{det}\left(J_{E_{e 1}}\right) & =Z_{e 1}\left[-P_{e 1}\left[\frac{r_{1}}{m+P_{e 1}}\left(1-\frac{P_{e 1}}{K_{1}}\right)-\frac{r_{1}}{K_{1}}\right] \frac{r_{2}}{K_{2}}+\frac{\beta_{2} K_{2}}{\left(m+P_{e 1}\right)^{2}} \frac{\beta_{1} P_{e 1}}{m+P_{e 1}}\right] \\
& <\frac{Z_{e 1} r_{1} r_{2} P_{e 1}}{m K_{1} K_{2}\left(m+P_{e 1}\right)}\left[\left(m-K_{2}\right)\left(m+2 P_{e 1}-K_{1}\right)\right] .
\end{aligned}
$$

Thus, if $m \geq K_{2}$, then $\operatorname{det}\left(J_{E_{e 1}}\right)<0$.

Hence, $E_{e 1}=\left(P_{e 1}, Z_{e 1}\right)$ is a saddle point if $K_{2} \leq m$.

Lemma 2.3 If there exists one positive equilibrium $E_{e 3}=\left(P_{e 3}, Z_{e 3}\right)$ for system $(1.1)$ and $P_{e 3}<$ $\frac{K_{1}-m}{2}$, then the equilibrium $E_{e 3}=\left(P_{e 3}, Z_{e 3}\right)$ is locally asymptotically stable when $K_{2} \geq m$ and $\frac{r_{1}}{r_{2}+\beta_{2}}<\frac{K_{1}}{K_{1}-m}$, or $K_{2} \geq m$ and $\left(r_{1} K_{1}-r_{1} m-r_{2} K_{1}-K_{1} \beta_{2}\right)^{2}-8 r_{1} r_{2} K_{1} m<0$.

Proof In the same manner, we define

$$
F(P)=-\frac{r_{1}}{K_{1} \beta_{1}}\left[P^{2}+\left(m-K_{1}\right) P-K_{1} m\right]-\left[K_{2}+\frac{\beta_{2} K_{2} P}{r_{2}(m+P)}\right] .
$$

Under the conditions of (ii), we can find that

$$
F^{\prime}(P)<0 \quad\left(0<P<\frac{K_{1}-m}{2}\right) .
$$

Thus

$$
\frac{\beta_{1} \beta_{2} K_{2}}{(m+P)^{2}}>-\frac{r_{1} r_{2}}{m K_{1}}\left(m+2 P-K_{1}\right) \quad\left(0<P<\frac{K_{1}-m}{2}\right) .
$$

Therefore

$$
\operatorname{det}\left(J_{E_{e 3}}\right)>\frac{Z_{e 3} r_{1} r_{2} P_{e 3}}{m K_{1} K_{2}\left(m+P_{e 3}\right)}\left[\left(m-K_{2}\right)\left(m+2 P_{e 3}-K_{1}\right)\right],
$$

when $m \geq K_{2}$, we obtain $\operatorname{det}\left(J_{E_{e 1}}\right)>0$.

Here

$$
\begin{aligned}
\operatorname{tr}\left(J_{E_{e 3}}\right)= & P_{e 3}\left[\frac{r_{1}}{m+P_{e 3}}\left(1-\frac{P_{e 3}}{K_{1}}\right)-\frac{r_{1}}{K_{1}}\right]-\frac{r_{2} Z_{e 3}}{K_{2}} \\
= & \frac{-2 r_{1} P_{e 3}^{2}+\left(r_{1} K_{1}-r_{1} m-r_{2} K_{1}-K_{1} \beta_{2}\right) P_{e 3}-r_{2} K_{1} m}{K_{1}\left(m+P_{e 3}\right)} \\
& \left(0<P_{e 3}<\frac{K_{1}-m}{2}\right) .
\end{aligned}
$$

When

$$
\frac{r_{1}}{r_{2}+\beta_{2}}<\frac{K_{1}}{K_{1}-m}
$$

or

$$
\left(r_{1} K_{1}-r_{1} m-r_{2} K_{1}-K_{1} \beta_{2}\right)^{2}-8 r_{1} r_{2} K_{1} m<0
$$


then $\operatorname{tr}\left(J_{E_{e 3}}\right)<0$.

Hence, according to the above conditions, $E_{e 3}=\left(P_{e 3}, Z_{e 3}\right)$ is locally asymptotically stable.

Theorem 2.1 The positive equilibrium $E_{e 4}=\left(P_{e 4}, Z_{e 4}\right)\left(P_{e 4}>\frac{K_{1}-m}{2}\right)$ is globally asymptotically stable in the presence and the absence of diffusion if $0<K_{1} \leq \frac{r_{1} m\left(m+P_{e 4}\right)}{\beta_{1} Z_{e 4}}$ holds.

Proof Define a Lyapunov function

$$
V_{1}(P, Z)=\int_{P_{e 4}}^{P} \frac{X-P_{e 4}}{X} d X+\frac{\beta_{1}}{\beta_{2}} \int_{Z_{e 4}}^{Z} \frac{Y-Z_{e 4}}{Y} d Y
$$

We note that $V_{1}(P, Z)$ is nonnegative and $V_{1}(P, Z)=0$ if and only if

$$
(P(t), Z(t))=\left(P_{e 4}, Z_{e 4}\right) .
$$

Furthermore,

$$
\frac{d V_{1}}{d t}=\frac{P-P_{e 4}}{P} \frac{d P}{d t}+\frac{\beta_{1}}{\beta_{2}} \frac{Z-Z_{e 4}}{Z} \frac{d Z}{d t}
$$

By substituting the expressions for $\frac{d P}{d t}$ and $\frac{d Z}{d t}$ from system (1.1) in the absence of diffusion, we obtain

$$
\frac{d V_{1}}{d t}=\left(P-P_{e 4}\right)\left(r_{1}\left(1-\frac{P}{K_{1}}\right)-\frac{\beta_{1} Z}{m+P}\right)+\frac{\beta_{1}}{\beta_{2}}\left(Z-Z_{e 4}\right) r_{2}\left(1-\frac{Z}{K_{2}}\right)+\frac{\beta_{2} P}{m+P} .
$$

Using the condition that

$$
r_{1}\left(1-\frac{P_{e 4}}{K_{1}}\right)-\frac{\beta_{1} Z_{e 4}}{m+P_{e 4}}=0, r_{2}\left(1-\frac{Z_{e 4}}{K_{2}}\right)+\frac{\beta_{2} P_{e 4}}{m+P_{e 4}}=0
$$

(2.3) can be simplified as

$$
\frac{d V_{1}}{d t}=\left(-\frac{r_{1}}{K_{1}}+\frac{\beta_{1} Z_{e 4}}{\left(m+P_{e 4}\right)(m+P)}\right)\left(P-P_{e 4}\right)^{2}+\frac{\beta_{1} P_{e 4}}{\left(m+P_{e 4}\right)\left(K_{2}-Z_{e 4}\right)}\left(Z-Z_{e 4}\right)^{2} .
$$

Because $Z_{e 4}>K_{2}$, we have

$$
\frac{\beta_{1} P_{e 4}}{\left(m+P_{e 4}\right)\left(K_{2}-Z_{e 4}\right)}\left(Z-Z_{e 4}\right)^{2}<0
$$

and $\left(-\frac{r_{1}}{K_{1}}+\frac{\beta_{1} Z_{e 4}}{\left(m+P_{e 4}\right)(m+P)}\right)\left(P-P_{e 4}\right)^{2} \leq 0$ always occurs when

$$
0<K_{1} \leq \frac{r_{1} m\left(m+P_{e 4}\right)}{\beta_{1} Z_{e 4}}
$$

Therefore, $\frac{d V_{1}}{d t}<0$ if $0<K_{1} \leq \frac{r_{1} m\left(m+P_{e 4}\right)}{\beta_{1} Z_{e 4}}$ holds. 


\section{Stability and instability of the system with diffusion}

In this section, we consider how the diffusion affects the stability of system (1.2). The stability and instability of system (1.2) are considered as follows.

Let $0=u_{0}<u_{1}<u_{2}<\cdots<\infty\left(\lim _{i \rightarrow \infty} u_{i}=\infty\right)$ be the eigenvalues of $-\nabla^{2}$ on $\Omega$ with the zero-flux boundary condition. Set

$$
X:=\left\{U=(P, Z) \in\left[C^{1}(\Omega)\right]^{2}: \frac{\partial P}{\partial n}=\frac{\partial Z}{\partial n}=0\right\},
$$

and we then consider that $X=\bigoplus_{i=0}^{\infty} X_{i}$, where $X_{i}$ is the eigenspace corresponding to $u_{i}$.

Theorem 3.1 The positive equilibrium $E_{e 2}=\left(P_{e 2}, Z_{e 2}\right)$ in system (1.2) is uniformly asymptotically stable in the presence of diffusion.

Proof The linearization of system (1.2) at the positive equilibrium $E_{e 2}=\left(P_{e 2}, Z_{e 2}\right)$ is

$$
\begin{aligned}
& \frac{\partial}{\partial t}\left(\begin{array}{l}
P \\
Z
\end{array}\right)=\Gamma\left(\begin{array}{l}
P \\
Z
\end{array}\right)+\left(\begin{array}{l}
f_{1}\left(P-P_{e 2}, Z-Z_{e 2}\right) \\
f_{2}\left(P-P_{e 2}, Z-Z_{e 2}\right)
\end{array}\right), \\
& \Gamma=\left(\begin{array}{cc}
P_{e 2}\left[\frac{r_{1}}{m+P_{e 2}}\left(1-\frac{P_{e 2}}{K_{1}}\right)-\frac{r_{1}}{K_{1}}\right]+D_{1} \Delta & -\frac{\beta_{1} P_{e 2}}{m+P_{e 2}} \\
\frac{\beta_{2} K_{2} Z_{e 2}}{\left(m+P_{e 2}\right)^{2}} & -\frac{r_{2} Z_{e 2}}{K_{2}}+D_{2} \Delta
\end{array}\right) \\
&=\left(\begin{array}{cc}
\Gamma_{11}+D_{1} \Delta & \Gamma_{12} \\
\Gamma_{21} & \Gamma_{22}+D_{2} \Delta
\end{array}\right),
\end{aligned}
$$

where $f_{n}\left(\varepsilon_{1}, \varepsilon_{2}\right)=O\left(\varepsilon_{1}^{2}+\varepsilon_{2}^{2}\right)(n=1,2)$.

For each $i(i=0,1,2, \ldots), X_{i}$ is invariant under the operator $\Gamma$, and $u_{i}$ is an eigenvalue of $\Gamma$ on $X_{i}$ if and only if $\lambda_{i}$ is an eigenvalue of the matrix

$$
H_{i}=\left(\begin{array}{cc}
\Gamma_{11}-D_{1} u_{i} & \Gamma_{12} \\
\Gamma_{21} & \Gamma_{22}-D_{2} u_{i}
\end{array}\right) .
$$

Note that

$$
\begin{aligned}
& \operatorname{tr}\left(H_{i}\right)=\Gamma_{11}+\Gamma_{22}-\left(D_{1}+D_{2}\right) u_{i}, \\
& \operatorname{det}\left(H_{i}\right)=\Gamma_{11} \Gamma_{22}-\Gamma_{12} \Gamma_{21}+D_{1} D_{2} u_{i}^{2}-u_{i}\left(D_{2} \Gamma_{11}+D_{1} \Gamma_{22}\right) .
\end{aligned}
$$

It is obvious that $\operatorname{tr}\left(H_{i}\right)<0, \operatorname{det}\left(H_{i}\right)>0$. and the two eigenvalues $\lambda_{i}^{+}$and $\lambda_{i}^{-}$have negative real parts. For any $i \geq 0$, we can get the following.

If $\left(\operatorname{tr}\left(H_{i}\right)\right)^{2}-4 \operatorname{det}\left(H_{i}\right) \leq 0$, then $\operatorname{Re}\left(\lambda_{i}^{ \pm}\right)=\frac{1}{2} \operatorname{tr}\left(H_{i}\right) \leq \frac{1}{2} \Gamma_{11}<0$; and if $\left(\operatorname{tr}\left(H_{i}\right)\right)^{2}-4 \operatorname{det}\left(H_{i}\right)>$ 0 , since $\operatorname{tr}\left(H_{i}\right)<0$ and $\operatorname{det}\left(H_{i}\right)>0$, we have

$$
\begin{aligned}
\operatorname{Re}\left(\lambda_{i}^{-}\right) & =\frac{\operatorname{tr}\left(H_{i}\right)-\sqrt{\left(\operatorname{tr}\left(H_{i}\right)\right)^{2}-4 \operatorname{det}\left(H_{i}\right)}}{2} \leq \frac{1}{2} \operatorname{tr}\left(H_{i}\right) \leq \frac{1}{2} \Gamma_{11}<0, \\
\operatorname{Re}\left(\lambda_{i}^{+}\right) & =\frac{\operatorname{tr}\left(H_{i}\right)+\sqrt{\left(\operatorname{tr}\left(H_{i}\right)\right)^{2}-4 \operatorname{det}\left(H_{i}\right)}}{2} \\
& =\frac{2 \operatorname{det}\left(H_{i}\right)}{\operatorname{tr}\left(H_{i}\right)-\sqrt{\left(\operatorname{tr}\left(H_{i}\right)\right)^{2}-4 \operatorname{det}\left(H_{i}\right)}} \leq \frac{\operatorname{det}\left(H_{i}\right)}{\operatorname{tr}\left(H_{i}\right)}<C,
\end{aligned}
$$

where $C$ is dependent on $i$. 
Thus, a negative constant $C$ exists, which is independent of $i$, such that $\operatorname{Re}\left(\lambda_{i}^{ \pm}\right)<C$ for any $i$. By referring to [35], we find that the positive equilibrium $E_{e 2}=\left(P_{e 2}, Z_{e 2}\right)$ of system (1.2) is uniformly asymptotically stable in the presence of diffusion. The proof is complete.

Theorem 3.2 If there exists one positive equilibrium $E_{e 4}=\left(P_{e 4}, Z_{e 4}\right)$ in system (1.2) and $P_{e 4}>\frac{K_{1}-m}{2}$, then $E_{e 4}=\left(P_{e 4}, Z_{e 4}\right)$ is globally asymptotically stable in the presence of diffusion if $0<K_{1} \leq \frac{r_{1} m\left(m+P_{e 4}\right)}{\beta_{1} Z_{e 4}}$ holds.

Proof By referring to [36, 37] and the proof of Theorem 2.1, define

$$
V_{2}=\iint_{\Omega} V_{1}(P, Z) d \Omega
$$

Then by differentiating $V_{2}$ with respect to time $t$ along the solutions of system (1.2), we can obtain

$$
\frac{d V_{2}}{d t}=\iint_{\Omega} \frac{d V_{1}}{d t} d \Omega+\iint_{\Omega}\left(D_{1} \frac{\partial V_{1}}{\partial P} \Delta P+D_{2} \frac{\partial V_{1}}{\partial Z} \Delta Z\right) d \Omega
$$

Using Green's first identity in the plane, we obtain

$$
\begin{aligned}
\frac{d V_{2}}{d t}= & \iint_{\Omega} \frac{d V_{1}}{d t} d \Omega-D_{1} \iint_{\Omega} \frac{\partial^{2} V_{1}}{\partial P^{2}}\left(\left(\frac{\partial P}{\partial x}\right)^{2}+\left(\frac{\partial P}{\partial y}\right)^{2}\right) d \Omega \\
& -D_{2} \iint_{\Omega} \frac{\partial^{2} V_{1}}{\partial Z^{2}}\left(\left(\frac{\partial Z}{\partial x}\right)^{2}+\left(\frac{\partial Z}{\partial y}\right)^{2}\right) d \Omega
\end{aligned}
$$

where

$$
\frac{\partial^{2} V_{1}}{\partial P^{2}}=\frac{P_{e 4}}{P^{2}}>0 \quad \text { and } \quad \frac{\partial^{2} V_{1}}{\partial Z^{2}}=\frac{\beta_{1} Z_{e 4}}{\beta_{2} Z^{2}}>0
$$

Based on the above analysis, if

$$
0<K_{1} \leq \frac{r_{1} m\left(m+P_{e 4}\right)}{\beta_{1} Z_{e 4}}
$$

then $\frac{d V_{1}}{d t}<0$, and thus $\frac{d V_{2}}{d t}<0$.

This completes the proof.

Next, we analyze the formation of patterns. Based on the analysis above, we know that the positive equilibrium point $E_{e 3}=\left(P_{e 3}, Z_{e 3}\right)$ of system (1.2) is locally asymptotically stable when $K_{2} \geq m$ and $\frac{r_{1}}{r_{2}+\beta_{2}}<\frac{K_{1}}{K_{1}-m}$, or $K_{2} \geq m$ and $\left(r_{1} K_{1}-r_{1} m-r_{2} K_{1}-K_{1} \beta_{2}\right)^{2}-8 r_{1} r_{2} K_{1} m<0$ holds. In this case, we can reach the following conclusion.

Theorem 3.3 If the positive equilibrium $E_{e 3}=\left(P_{e 3}, Z_{e 3}\right)$ is locally asymptotically stable in system (1.1), then for system (1.2), the equilibrium $E_{e 3}=\left(P_{e 3}, Z_{e 3}\right)$ is stable when

$$
\frac{D_{2}}{D_{1}}<\frac{-\left(2 a_{12} a_{21}-a_{11} a_{22}\right)+2 \sqrt{a_{12}^{2} a_{21}^{2}-a_{11} a_{22} a_{12} a_{21}}}{a_{11}^{2}}
$$


and the criterion for Turing instability for system (1.2) satisfies the following condition:

$$
\frac{D_{2}}{D_{1}}>\frac{-\left(2 a_{12} a_{21}-a_{11} a_{22}\right)+2 \sqrt{a_{12}^{2} a_{21}^{2}-a_{11} a_{22} a_{12} a_{21}}}{a_{11}^{2}},
$$

where

$$
J_{E_{e 3}}=\left(\begin{array}{ll}
a_{11} & a_{12} \\
a_{21} & a_{22}
\end{array}\right)
$$

is the Jacobian matrix at $E_{e 3}=\left(P_{e 3}, Z_{e 3}\right)$.

Proof The linearized form of (1.2) that corresponds to the equilibrium state $E_{e 3}=\left(P_{e 3}, Z_{e 3}\right)$ is given by

$$
\left\{\begin{array}{l}
\frac{\partial p}{\partial t}=a_{11} p+a_{12} z+D_{1} \frac{\partial^{2} p}{\partial s^{2}}, \\
\frac{\partial z}{\partial t}=a_{21} p+a_{22} z+D_{2} \frac{\partial^{2} z}{\partial s^{2}},
\end{array}\right.
$$

where $P=P_{e 3}+p, Z=Z_{e 3}+z$, and $(p, z)$ are small perturbations of $(P, Z)$ about the equilibrium $E_{e 3}=\left(P_{e 3}, Z_{e 3}\right)$. We expand the solution of system (1.2) into a Fourier series:

$$
\left(\begin{array}{l}
p \\
z
\end{array}\right)=\sum_{k}\left(\begin{array}{l}
C_{k}^{1} \\
C_{k}^{2}
\end{array}\right) e^{\lambda_{k} t+i k s}
$$

where $k$ is the wave number of the solution. By combining (3.4) and (3.5), we obtain

$$
\lambda_{k}\left(\begin{array}{l}
C_{k}^{1} \\
C_{k}^{2}
\end{array}\right)=\left(\begin{array}{cc}
a_{11}-k^{2} D_{1} & a_{12} \\
a_{21} & a_{22}-k^{2} D_{2}
\end{array}\right)\left(\begin{array}{c}
C_{k}^{1} \\
C_{k}^{2}
\end{array}\right) .
$$

Hence, we can obtain the characteristic equation as follows:

$$
\lambda^{2}-\operatorname{tr}_{k} \lambda_{k}+\Delta_{k}=0
$$

where

$$
\begin{aligned}
& \operatorname{tr}_{k}=a_{11}+a_{22}-k^{2}\left(D_{1}+D_{2}\right), \\
& \Delta_{k}=\operatorname{det}\left(J_{E_{e 3}}\right)-k^{2}\left(a_{11} D_{2}+a_{22} D_{1}\right)+k^{4} D_{1} D_{2} .
\end{aligned}
$$

In order to allow the system to reach Turing instability, at least one of the following conditions must be satisfied: $\operatorname{tr}_{k}>0$ and $\Delta_{k}<0$. However, it is obvious that $\operatorname{tr}_{k}<0$. Thus only the condition $\Delta_{k}<0$ can give rise to instability. Therefore, a necessary condition for the system to be unstable is that

$$
\operatorname{det}\left(J_{E_{e 3}}\right)-k^{2}\left(a_{11} D_{2}+a_{22} D_{1}\right)+k^{4} D_{1} D_{2}<0 .
$$

We define

$$
F\left(k^{2}\right)=k^{4} D_{1} D_{2}-k^{2}\left(a_{11} D_{2}+a_{22} D_{1}\right)+\operatorname{det}\left(J_{E_{e 3}}\right) .
$$


If we let $k_{\min }^{2}$ be the corresponding value of $k^{2}$ for the minimum value of $F\left(k^{2}\right)$, then

$$
k_{\min }^{2}=\frac{a_{11} D_{2}+a_{22} D_{1}}{2 D_{1} D_{2}}>0 .
$$

Thus, the corresponding minimum value of $F\left(k^{2}\right)$ is

$$
F\left(k_{\min }^{2}\right)=\operatorname{det}\left(J_{E_{e 3}}\right)-\frac{\left(a_{11} D_{2}+a_{22} D_{1}\right)^{2}}{4 D_{1} D_{2}} .
$$

If we let $F\left(k_{\min }^{2}\right)<0$, the sufficient condition for the system to be unstable reduces to

$$
\frac{D_{2}}{D_{1}} a_{11}+a_{22}>2 \sqrt{\operatorname{det}\left(J_{E_{e 3}}\right) \frac{D_{2}}{D_{1}}}
$$

which yields

$$
\frac{D_{2}}{D_{1}}>\frac{-\left(2 a_{12} a_{21}-a_{11} a_{22}\right)+2 \sqrt{a_{12}^{2} a_{21}^{2}-a_{11} a_{22} a_{12} a_{21}}}{a_{11}^{2}} .
$$

In Theorem 3.3, we found that (3.7) is a sufficient condition for the diffusive instability of system (1.2). If criterion (3.7) is satisfied, the diffusion of the plankton population can cause the system to shift from a stable state to an unstable state. In ecological terms, this means that if the zooplankton diffusion rate is higher than the phytoplankton diffusion rate and (3.7) is satisfied, then the instability will continue to prevail. At this time point, we can use the system parameters to obtain the patterns of the plankton populations. The patterns of the plankton populations are considered in Section 4.

\section{Numerical results}

\subsection{Numerical analysis}

In this section, we will explore the carrying capacity of zooplankton $K_{2}$ and the half saturation constant $m$ and the influence on the steady-state transformation problem of the proposed system (1.1) and (1.2) using a numerical simulation analysis; thus, some parameters are fixed as follows: $r_{1}=0.8, K_{1}=1, \beta_{1}=0.8, \beta_{2}=0.7, r_{2}=0.7$.

Figure 1A describes a double-parameter bifurcation diagram, which shows how the parameters $K_{2}$ and $m$ affect the stability of system (1.1). It is easy to find from Figure 1A

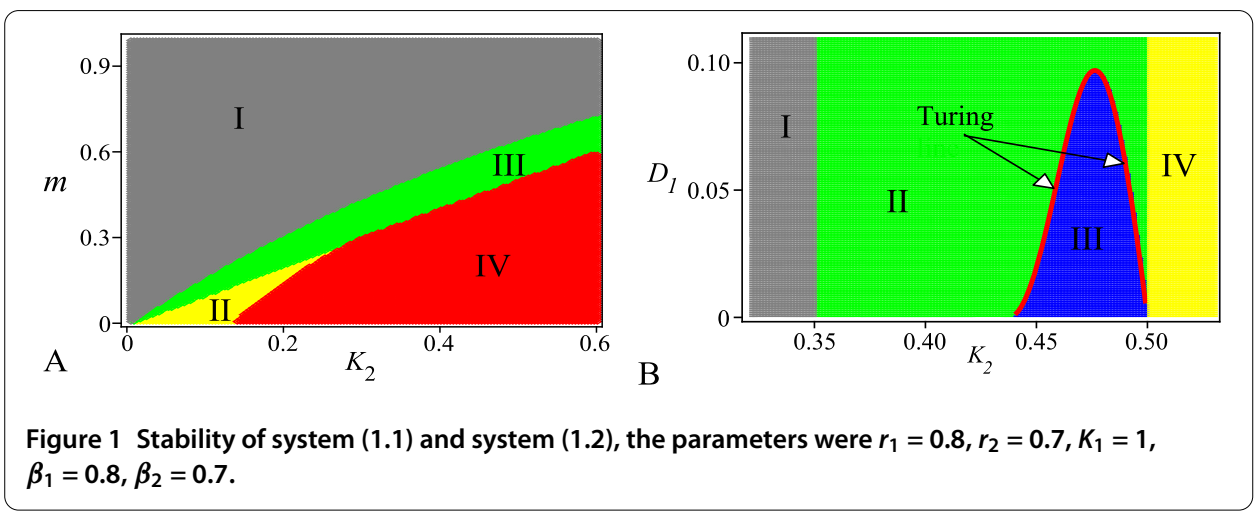



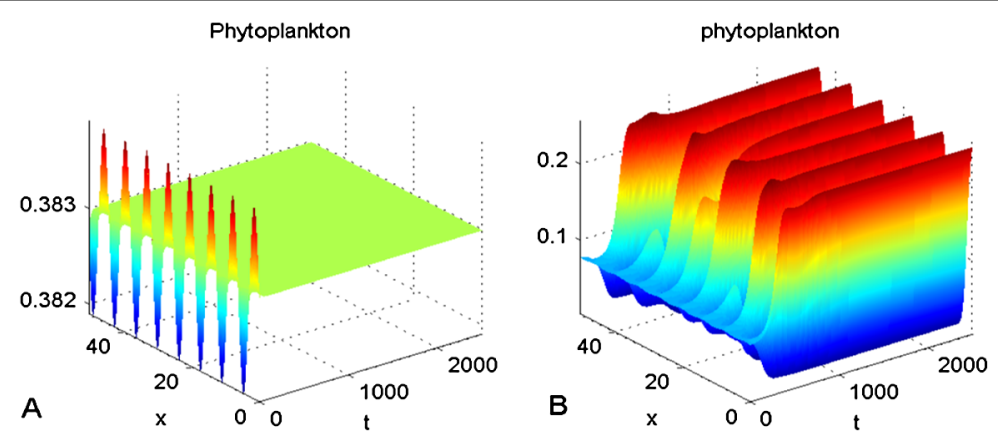

Figure 2 Numerical stability of system (1.2) for $r_{1}=0.8, r_{2}=0.7, K_{1}=1, \beta_{1}=0.8, \beta_{2}=0.7, m=0.5$, $D_{1}=\mathbf{0 . 0 2} . \mathbf{A}: K_{2}=0.38 ; \mathbf{B}: K_{2}=0.47$.

that system (1.1) is globally asymptotically stable in the gray area (I) and is locally asymptotically stable in the green area (III). Nevertheless, system (1.1) has two equilibria in the yellow area (II), one is locally asymptotically stable and the other is unstable. But it is a pity that system (1.1) does not have a positive equilibrium in the red area (IV). These results are consistent with the results in Section 2, which suggested the correctness and feasibility of the theoretical deduction.

In order to comprehensively analyze the parameter $K_{2}$ and $D_{1}$ and the influence on the stability of system (1.2), we set $r_{1}=0.8, K_{1}=1, \beta_{1}=0.8, \beta_{2}=0.7, r_{2}=0.7, m=0.5$. On setting $D_{2}=10$, the Turing bifurcation curve can be obtained, as is shown in Figure 1B. Now, it is worthwhile to point out from Figure 1B that system (1.2) is globally asymptotically stable in the gray area (I), locally asymptotically stable in the green area (II), and unstable in the blue area (III), respectively. Moreover, it should be stressed that the red curve indicates the Turing bifurcation curves in system (1.2), and the yellow area in which there is no positive equilibrium. According to the premise $D_{1}<D_{2}$, we can determine the range of the parameter $K_{2}$ in the region III in Figure 1B; system (1.2) may shift from a stable state to an unstable state when we adjust the size of $D_{1}$, so that system (1.2) can reach Turing instability in the whole of area III, which also verifies the conclusion of Theorem 3.3. Further, on setting the range of the parameter $D_{1}$ in the region III, system (1.2) can also shift from a stable state to an unstable state when we adjust the size of $K_{2}$. Based on the above results, it is worth to point out that the parameters $K_{2}$ and $D_{1}$ are sensitive in the steadystate transformation problem of system (1.2). Furthermore, it is evident from Figure 2 that the considered equilibrium is stable when $K_{2}=0.38$ and is unstable when $K_{2}=0.47$. These results suggested that system (1.2) has a dynamic conversion process from the steady-state solutions to the nonstationary solutions with varying parameter $K_{2}$, which can provide a simulation verification to further elaborate the sensitiveness of the environmental carrying capacity $K_{2}$ in terms of the steady-state transformation.

\subsection{Numerical simulations}

In order to further expound the environmental carrying capacity $K_{2}$ and the diffusion $D_{1}$ affecting the spatial distributions of phytoplankton population, some kinds of patterns will be given using the numerical simulations. Here, we perform extensive numerical simulations of system (1.2) in two dimensional space with the same parameter values as given in Figure 1B. Meanwhile, the value range of the parameters $K_{2}$ and $D_{1}$ can be chosen in the area III in Figure 1B. 

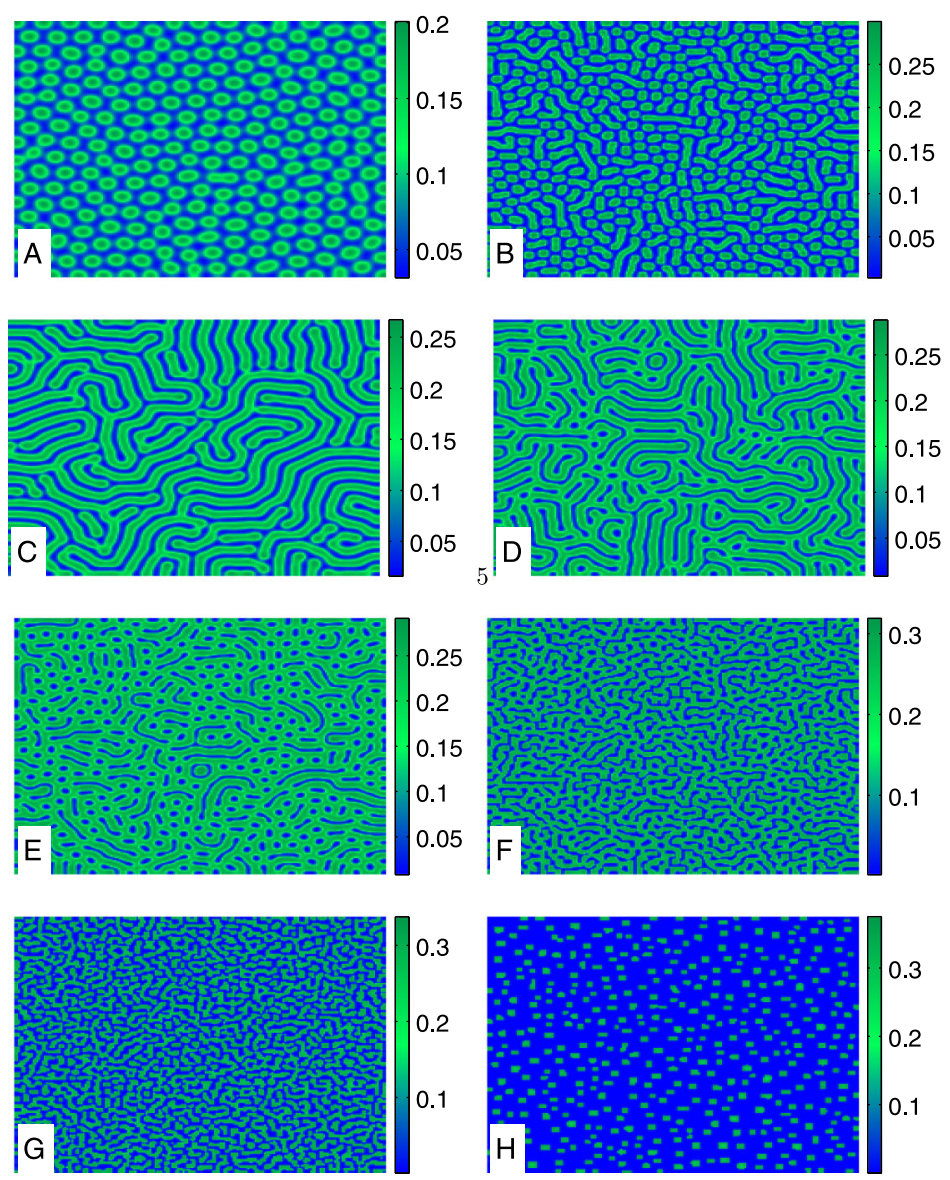

Figure 3 Eight categories of patterns obtained with system (1.2) at $t=50,000$, where the parameters were $r_{1}=\mathbf{0 . 8}, \boldsymbol{r}_{2}=\mathbf{0 . 7}, K_{1}=\mathbf{1}, \boldsymbol{\beta}_{\mathbf{1}}=\mathbf{0 . 8}, \boldsymbol{\beta}_{\mathbf{2}}=\mathbf{0 . 7}, \boldsymbol{m}=\mathbf{0 . 5}, \boldsymbol{D}_{\mathbf{2}}=\mathbf{1 0}$. A: $K_{2}=0.466, D_{1}=0.061 ; \mathrm{B}: K_{2}=0.466$ $D_{1}=0.015 ; \mathbf{C}: K_{2}=0.457, D_{1}=0.031 ; \mathbf{D}: K_{2}=0.45, D_{1}=0.019 ; \mathbf{E}: K_{2}=0.446, D_{1}=0.016 ; \mathbf{F}: K_{2}=0.45, D_{1}=0.007 ;$ G: $K_{2}=0.46, D_{1}=0.005 ; \mathbf{H}: K_{2}=0.495, D_{1}=0.003$.

It is well known that Figure 3A is a hot-spot pattern, which comprises green spots on a blue background. Furthermore, it is also illustrated from Figure 3A that the hot spots are isolated zones with high phytoplankton densities, where the zooplankton population has a low density. However, with the change of parameter value, the spatial distributions of the phytoplankton population will have a series of dynamic evolutionary processes. Figure 3B is a hot-spot-stripes pattern, with small hot spots that can form stripes. Figure $3 \mathrm{C}$ is a striped pattern, which required a long time to settle down and we obtained only stripes. Figure 3D is similar to Figure 3E, where both are cold-spot-stripes patterns, i.e., the cold spots are isolated zones with low phytoplankton densities. However, Figure 3D can contain more stripes than Figure 3E, and the stripes are much longer. Figure 3F and Figure 3G show more complex striped patterns compared with Figure 3C. In Figure 3F, the distribution of stripes and spots is highly irregular, and the density of phytoplankton is low in these stripes and isolated spots. In Figure 3G, the distribution of stripes is more cluttered and similar to a maze. In the stripes shown in Figure 3G, the density of phytoplankton is lower than that in Figure 3F. Figure 3H shows a more interesting hot-spot pattern produced by system (1.2). Although Figures $3 \mathrm{H}$ and $3 \mathrm{~A}$ are both spot pattern; they have an obvious difference in the form. Stated succinctly, it is worthy of emphasis that rich patterns in 


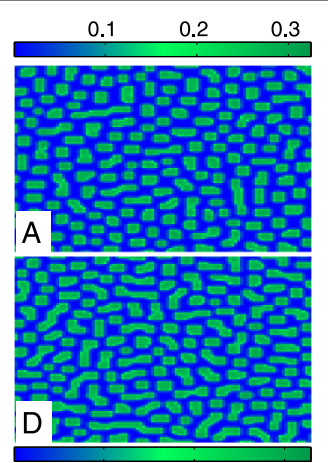

0.1
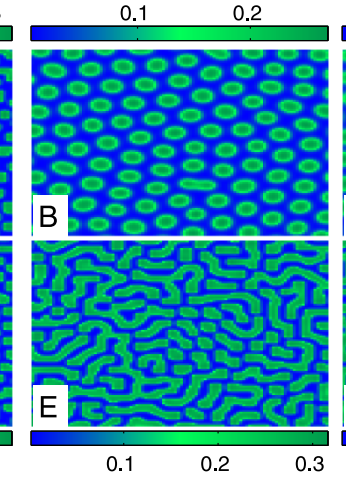

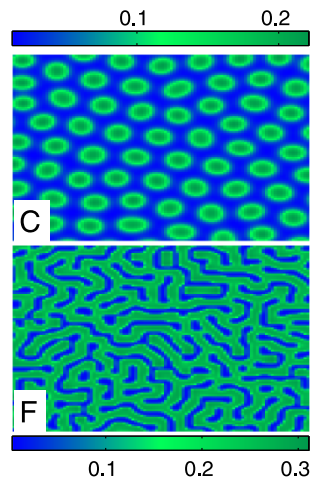

Figure 4 Changes in the patterns with different values of $K_{2}$ or $D_{1}$ at $t=50,000$, where the parameters were $\mathrm{A}: K_{2}=0.475, D_{1}=0.01 ; \mathrm{B}: K_{2}=0.475, D_{1}=0.03 ; \mathrm{C}: K_{2}=0.475, D_{1}=0.05$; D: $K_{2}=0.47, D_{1}=0.01 ; \mathrm{E}: K_{2}=0.46, D_{1}=0.01 ; \mathrm{F}: K_{2}=0.45, D_{1}=0.01$.

Figure 3 can be obtained by controlling the value of the environmental carrying capacity $K_{2}$ and the diffusion $D_{1}$, where the patterns illustrate the complex spatial distribution of phytoplankton in system (1.2).

In order to deeply inquire into the parameters $D_{1}$ and $K_{2}$ affecting the spatial distributions of phytoplankton population, a series of patterns will be displayed. It is worth to point out from Figures $4 \mathrm{~A}-4 \mathrm{C}$ with $K_{2}=0.475$ that system (1.2) has a hot-spot pattern at $D_{1}=0.01,0.03,0.05$, but the spots in Figure $4 \mathrm{~B}$ are elliptical and the spots in Figure $4 \mathrm{C}$ are bigger than the other two. These results suggested that the spatial distribution of phytoplankton population will gradually become larger with increase of the value of $D_{1}$. It should also be stressed from Figures 4D-4E with $D_{1}=0.01$ that the spatial distribution of phytoplankton will gradually change from spots to spot stripes and finally stripes with the decrease of $K_{2}$. These results illustrated that the parameters $K_{2}$ and $D_{1}$ have an inherent ability to influence the spatial distribution of phytoplankton. Stated succinctly, it must be emphasized that the diffusion and the environmental carrying capacity have an important role to play in the spatial distribution of phytoplankton.

The above numerical results have shown some dynamics in system (1.2), and it is found that there exist abundant patterns in system (1.2). In order to further reveal the spatial distribution of phytoplankton, another parameter set is employed, as follows: $r_{1}=2.425$, $K_{1}=1, \beta_{1}=1.35$ [38], $\beta_{2}=1.0125, r_{2}=0.97, m=0.35$, where the parameter $\beta_{2}$ was derived from the literature $[38,39]$, and the parameters $r_{1}$ and $r_{2}$ were derived from the literature [40]. The other parameters (i.e., $k_{2}, D_{1}$, and $D_{2}$ ) were chosen as controlling parameters.

We perform a numerical simulation of system (1.2) under the above parameter set, and the results are shown in Figure 5. It is shown from Figure 5 that there exist four kinds of pattern formations at least in system (1.2) based on this parameter set when the parameters $k_{2}, D_{1}$, and $D_{2}$ change. By comparison, it can be found that the results shown by Figure 5 are similar to the patterns shown by Figure 3, their distribution of spatial structure are coincident, for example, the spatial structure shown by Figure 5A agrees with the one shown by Figure 3D; and Figure 5B is similar to Figure 3E; the patterns shown by Figures $5 \mathrm{C}$ and $5 \mathrm{D}$ are coincident with the ones shown by Figures $3 \mathrm{G}$ and $3 \mathrm{H}$, respectively. These results suggest that there may be abundant patterns in the spatial distribution of phytoplankton. 

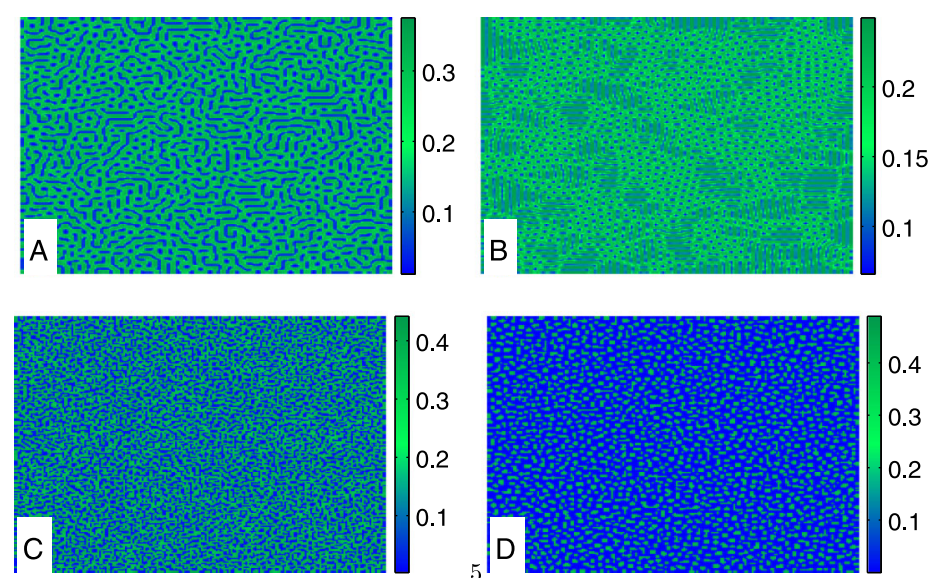

Figure 5 Four categories of patterns obtained with system (1.2) at $t=50,000$, where the parameters were $r_{1}=\mathbf{2 . 4 2 5}, r_{2}=\mathbf{0 . 9 7}, K_{1}=1, \beta_{1}=1.35, \beta_{2}=1.0125, m=0.35, D_{2}=10 . \mathrm{A}: K_{2}=0.52, D_{1}=0.047$, $D_{2}=3 ; \mathbf{B}: K_{2}=0.52, D_{1}=0.047, D_{2}=1 ; \mathbf{C}: K_{2}=0.52, D_{1}=0.017, D_{2}=3 ; \mathbf{D}: K_{2}=0.55, D_{1}=0.015, D_{2}=3$.

\section{Discussion}

In this study, we studied a nonlinear dynamics of a phytoplankton-zooplankton system with diffusion in detail. The structure of the system is very simple, but the study showed that the composition of the positive equilibrium was highly complex in the system. For system (1.1), our theoretical results show that if $K_{1}>m$, the value of the environmental carrying capacity of the zooplankton will influence the dynamical behaviors of system (1.1). With the value of $K_{2}$ changing, the stability of system (1.1) will change. Our numerical simulation leads to the same conclusion, through the results of Figure 1A; if the value of $m$ is smaller, then it is obvious that the stability of system (1.1) may change with the increase of parameter $K_{2}$. The result can suggest that the environmental carrying of the zooplankton is an important critical factor to influence the system dynamics.

The reaction-diffusion system exhibits some interesting dynamics depending on the parameters of the system. One of the main aims in our study is to analyze the effect of diffusion on the properties, especially the stability of the system. However, the results of Theorems 3.1 and 3.2 show that diffusion did not influence the stability of the system in some situations. The theoretical results show that only if the value of $\frac{D_{2}}{D_{1}}$ grows large enough, then the equilibrium $E_{e 3}$ will shift from a stable state to an unstable state. We also show this result through a two dimensional bifurcation diagram (see Figure 1B). Our results show that the Turing instability will occur under certain conditions in the reaction-diffusion system. The occurrence of a Turing instability means that a solution which is stable in the absence of diffusion becomes unstable when diffusion is incorporated in the system. An effective way to study it is to give a small perturbation around the steady-state solutions and then obtain the conditions under which these fluctuations increase. In Figure 1B, our numerical results not only show that the diffusion can induce the occurrence of a Turing instability but also indicate that the environment carrying capacity of the zooplankton has an important role to play in the steady-state transformation.

Both theoretical and numerical analysis results have pointed out that the diffusion coefficient and the environment carrying capacity of the zooplankton have a significant impact on the dynamic properties. In 1993 [41], Pearson have presented 12 classic categories of patterns and these pattern formation have been studied further in recent years [42]. Our 
results show that the dynamics of system (1.2) comprised the diffusion controlled formation of stripes, hot/cold stripes/spots and some more interesting patterns. We aim to further demonstrate the influence of important parameters on the system by patterns formation; thus the results of Figure 4 are intuitive in reflecting the important role in these two parameters in the system. In general, the pattern formation can verify the rich dynamic properties around the Turing line of system, and the importance of the two parameters $K_{2}$ and $D_{1}$ is also deduced by the variation of the pattern.

Stated succinctly, the environment carrying capacity of the zooplankton and the diffusion term can seriously affect the dynamical behaviors of systems (1.1) and (1.2). Furthermore, it is our hope that these results can provide some reference value in the study of the internal operating rules and characteristics of marine ecosystems.

Competing interests

The authors declare that they have no competing interests.

\section{Authors' contributions}

All authors contributed equally and significantly in writing this article. All authors read and approved the final manuscript.

\section{Author details}

${ }^{1}$ School of Mathematics and Information Science, Wenzhou University, Wenzhou, Zhejiang 325035, P.R. China. ${ }^{2}$ Zhejiang Provincial Key Laboratory for Water Environment and Marine Biological Resources Protection, Wenzhou University, Wenzhou, Zhejiang 325035, P.R. China. ${ }^{3}$ School of Life and Environmental Science, Wenzhou University, Wenzhou, Zhejiang 325035, P.R. China.

\section{Acknowledgements}

This work was supported by the National Natural Science Foundation of China (Grant No. 31370381), the National Key Basic Research Program of China (973 Program, Grant No. 2012CB426510), and the Key Program of Zhejiang Provincial Natural Science Foundation of China (Grant No. LZ12C03001).

Received: 15 July 2015 Accepted: 8 August 2016 Published online: 23 August 2016

\section{References}

1. Anderson, D: Turning back the harmful red tide. Nature 338, 513-514 (1997)

2. Chattopadhyay, J, Sarkar, RR, El Abdllaoui, A: A delay differential equation model on harmful algal blooms in the presence of toxic substances. IMA J. Math. Appl. Med. Biol. 19, 137-161 (2002)

3. Morozov, AY, Petrovskii, SV, Nezlin, NP: Towards resolving the paradox of enrichment: the impact of zooplankton vertical migrations on plankton system stability. J. Theor. Biol. 248, 501-511 (2007)

4. Mukhopadhyay, B, Bhattacharya, R: A delay-diffusion model of marine plankton ecosystem exhibiting cyclic nature of blooms. J. Biol. Phys. 31, 3-22 (2005)

5. Yang, J, Zhao, M: Complex behavior in a fish algae consumption model with impulsive control strategy. Discrete Dyn. Nat. Soc. 2011, Article ID 163541 (2011)

6. Maiti, A, Jana, MM, Samanta, GP: Deterministic and stochastic analysis of a ratio-dependent of a prey-predator system with delay. Nonlinear Anal., Model. Control 12(3), 383-398 (2007)

7. Samanta, GP: Stochastic analysis of a prey-predator system. Int. J. Math. Educ. Sci. Technol. 25(6), 793-803 (1994)

8. Zhao, M, Wang, YZ, Chen, LS: Dynamic analysis of a predator-prey (pest) model with disease in prey and involving an impulsive control strategy. J. Appl. Math. 2012, Article ID 969425 (2012)

9. Dai, CJ, Zhao, M: Mathematic and dynamic analysis of a prey-predator system in the presence of alternative prey with impulsive state feedback control. Discrete Dyn. Nat. Soc. 2012, Article ID 724014 (2012)

10. Gazi, NH, Bandyopadyay, M: Effect of time delay on a detritus-based ecosystem. Int. J. Math. Math. Sci. 2006, Article ID $25619(2006)$

11. Fay, P: The Blue-Greens. Arnold, London (1983)

12. Sharma, A, Sharma, AK, Agnihotri, K: The dynamic of plankton-nutrient interaction with delay. Appl. Math. Comput. 231, 503-515 (2014)

13. Steele, JH: Structure of Marine Ecosystems. Blackwell Sci., Oxford (1974)

14. Beretta, E, Bischi, G, Solimano, F: Stability in chemostat equations with delayed nutrient recycling. J. Math. Biol. 28, 99-111 (1990)

15. Taylor, AJ: Characteristic properties of model for the vertical distributions of phytoplankton under stratification. Ecol. Model. 40, 175-199 (1988)

16. Wroblewski, JS, Sarmiento, JL, Flierl, GR: An ocean basin scale model of plankton dynamics in the North Atlantic: 1. Solutions for the climatological oceanographic condition in may. Glob. Biogeochem. Cycles 2, 199-218 (1988)

17. Janga, SRJ, Baglama, J, Rick, J: Nutrient-phytoplankton-zooplankton models with a toxin. Math. Comput. Model. 43, 105-118 (2006)

18. Hallegraeff, G: A review of harmful algae blooms and the apparent global increase. Phycologia 32, 79-99 (1993)

19. Chattopadhyay, J, Sarkar, R: A delay differential equation model on harmful algal blooms in the presence of toxic substances. IMA J. Math. Appl. Med. Biol. 19, 137-161 (2002) 
20. Keating, Kl: Algal Metabolite Influence on Bloom Sequence in Eutrophic Freshwater Ponds. Ecological Monograph Series (EPA-600/3-76-081). Government Printing Office, Washington (1976)

21. Kirk, K, Gilbert, J: Variation in herbivore response to chemical defenses: zooplankton foraging on toxic cynobacteria. Ecology 73, 2208-2217 (1992)

22. Roy, S: The coevolution of two phytoplankton species on a single resource: allelopathy as a pseudo-mixotrophy. Theor. Popul. Biol. 75, 68-75 (2009)

23. Jiao, JJ, Chen, LS: Dynamical analysis on a single population model with state-dependent impulsively unilateral diffusion between two patches. Adv. Differ. Equ. 2012, 155 (2012)

24. Zhao, M, Lv, SJ: Chaos in a three-species food chain model with a Beddington-DeAngelis functional response. Chaos Solitons Fractals 40(5), 2305-2316 (2009)

25. Turing, AM: The chemical basis of morphogenesis. Philos. Trans. R. Soc. Lond. B 237, 37-72 (1952)

26. Segal, LA, Jackson, JL: Dissipative structure: an explanation and ecological example. J. Theor. Biol. 37, 545-559 (1972)

27. Holling, CS: The functional response of predators to prey density and its role in mimicry and population regulation. Mem. Entomol. Soc. Can. 45, 3-60 (1965)

28. Jang, S, Baglama, J, Wu, L: Dynamics of phytoplankton-zooplankton systems with toxin producing phytoplankton. Appl. Math. Comput. 227, 717-740 (2014)

29. Wang, YZ, Zhao, M: Dynamic analysis of an impulsively controlled predator-prey model with Holling type IV functional response. Discrete Dyn. Nat. Soc. 2012, Article ID 141272 (2012)

30. Ivlev, VS: Experimental Ecology of the Feeding of Fishes. Yale University Press, New Haven (1961)

31. Gazi, NH: Dynamics of a marine plankton system: diffusive instability and pattern formation. Appl. Math. Comput. 218, 8895-8905 (2012)

32. Dai, CJ, Zhao, M, Chen, LS: Dynamic complexity of an Ivlev-type prey-predator system with impulsive state feedback control. J. Appl. Math. 2012, Article ID 534276 (2012)

33. Fussmann, GF, Ellner, SP, Shertzer, KW, Shertzer, NG: Crossing the Hopf bifurcation in a live predator-prey system. Science 17, 1358-1360 (2000)

34. van Baalen, M, Krrivan, V, van Rijn, P, Sabelis, M: Alternative food, switching predators, and the persistence of predator-prey systems. Am. Nat. 157, 512-524 (2001)

35. Henry, D: Geometric Theory of Semilinear Parabolic Equations. Springer, New York (1981)

36. Dubey, B, Hussain, J: Modelling the interaction of two biological species in polluted environment. J. Math. Anal. Appl. $246,58-79$ (2000)

37. Dubey, B, Kumari, N, Upadhyay, RK: Spatiotemporal pattern formation in a diffusive predator prey system: an analytical approach. J. Appl. Math. Comput. 31, 413-432 (2009)

38. Landry, MR, Constantinou, J, Kirshtein, J: Microzooplankton grazing in the central equatorial Pacific during February and August. Deep-Sea Res., Part 2, Top. Stud. Oceanogr. 42, 657-672 (1995)

39. Fasham, MJR: Variations in the seasonal cycle of biological production in subarctic oceans: a model sensitivity analysis. Deep-Sea Res., Part 1, Oceanogr. Res. Pap. 42, 1111-1149 (1995)

40. Doney, SC, Glover, DM, Najjar, RG: A new coupled one-dimensional biological-physical model for the upper ocean: applications to the JGOFS Bermuda Atlantic timeseries study (BATS) site. Deep-Sea Res., Part 2, Top. Stud. Oceanogr. 43, 591-624 (1996)

41. Pearson, JE: Complex patterns in a simple system. Science 261, 189-192 (1993)

42. Wang, WM, Lin, YZ, Zhang, L, Rao, F, Tan, YJ: Complex patterns in a predator-prey model with self and cross-diffusion. Commun. Nonlinear Sci. Numer. Simul. 16, 2006-2015 (2011)

\section{Submit your manuscript to a SpringerOpen ${ }^{\ominus}$ journal and benefit from:}

- Convenient online submission

Rigorous peer review

- Immediate publication on acceptance

- Open access: articles freely available online

- High visibility within the field

- Retaining the copyright to your article 